\title{
Intrasound Therapy Improves the Mechanical Properties and the Morphology of Tendons in Fluoroquinolone-Induced Achilles Tendinopathy
}

\section{${ }^{1}$ AIYEGBUSI, AI; ${ }^{2}$ AJIBOLA, OA; ${ }^{3}$ SAMUEL, TA; ${ }^{4}$ BALOGUN, OJ; ${ }^{5}$ DURU, FI}

\author{
${ }^{l}$ Department of Physiotherapy, College of Medicine,University of Lagos, Nigeria aaiyegbusi@unilag.edu.ng \\ ${ }^{2}$ Department of Systems Engineering, University of Lagos, Akoka, Nigeria Email: oajibola@ umilag.edu.ng \\ ${ }^{3}$ Department of Biochemistry, College of Medicine, University of Lagos, Nigeria Email: tsamuel@unilag.edu.ng \\ ${ }^{4}$ Department of Biomedical Engineering, College of Medicine, University of Lagos, Nigeria Email: obalogun@unilag.edu.ng \\ ${ }^{5}$ Department of Anatomy, College of Medicine, University of Lagos, Nigeria Email: fduru@ unilag.edu.ng \\ *Corresponding Author Email: aaiyegbusi@unilag.edu.ng; Tel: +2348023212513
}

\begin{abstract}
Fluoroquinolones (FQs), especially Pefloxacin (PEF) have been reported to increase risk of Achilles tendon rupture. However, prior studies showed that intrasound therapy (ITR) significantly improved the morphology of the healing tendon. This study aimed to compare the effects of 7,14 and 21 days intrasound treatment on the mechanical properties and morphology of the Achilles tendon in fifty (50) male Sprague-Dawley rats induced with FQ and randomized into three main categories designated as Normal control (A), Experimental control (B)and Treatment groups (C). All animals in groups B and C were orally administered PEF $400 \mathrm{mg} / \mathrm{kg}$ in a fixed volume of $2.5 \mathrm{ml} / \mathrm{kg}$ daily for 6 consecutive days. The Achilles tendon of both hind limbs in group C were treated with the lowest intensity ITR 5 minutes daily for 7, 14 and 21 consecutive days. All the animals were serially sacrificed on days 8, 15 and 22 and the tendons excised and processed. Data were analyzed with RSTUDIO version 4.0.02 and the level of significance set at 0.05. Kruskal Wallis showed no significant differences $(p>0.05)$ in all the biomechanical parameters across all the groups. Compared with group B, ITR 14 days treatment had the best improvement in all biomechanical variables, though insignificant ( $p>0.05)$. In the Intrasound groups, there were significant positive correlations of Nitric Oxide Synthase (NOS) with ultimate tensile stress and tensile load ( $\mathrm{p}=$ $0.005 ; \mathrm{p}=0.003$ ) respectively. Intrasound treatment to the Achilles tendon for 14 days improved the mechanical properties and morphology of tendons in Fluoroquinolone-induced tendinopathy.
\end{abstract}

DOI: https://dx.doi.org/10.4314/jasem.v25i7.27

Copyright: Copyright $\odot 2021$ Aiyegbusi. This is an open access article distributed under the Creative Commons Attribution License (CCL), which permits unrestricted use, distribution, and reproduction in any medium, provided the original work is properly cited.

Dates: Received: 10 May 2021; Revised: 28 June 2021; Accepted: 01 July 2021

Keywords: Fluoroquinolones, Pefloxacin, Intrasound therapy, Tendinopathy, Achilles tendon

Fluoroquinolones (FQs) are broad-spectrum antibiotics that have been reported to have very potent cytotoxic effects on human connective tissue (Aranha et al., 2000). Studies have shown they are responsible for a 3 to 6 times increased risk of developing tendinitis or rupture of the Achilles tendon in patients (Sode et al., 2007). In an evaluation of more than 11 000 patients, rates of 2.4 incidences per 10000 patient prescriptions for tendinitis and 1.2 per 10000 for tendon rupture were cited (Van der Linden et al., 2007). Recent studies have estimated the incidence of FQ-associated tendinopathy to be between $0.14 \%$ and $0.4 \%$. Mostly involving sporting or very physically active persons (Stephenson 2013; Budny \& Ley, 2015). Pefloxacin which is highly prescribed has been reported to be one of the FQs that induced the greatest number of lesions with the strongest toxic potential (Desbordes et al., 1993; Kashida \& Kato, 1997). One proposed mechanism of tendon injury is the accumulation of free radicals during the inflammatory process secondary to antibiotic use (Seeger et al., 2006). These substances are toxic to tendon cells and can lead to necrosis (Childs, 2007). With frequent use, there are histologic changes and disorganization of the extracellular matrix (ECM) leading to a remodeling process that weakens the tendon matrix eventually as demonstrated also in fluoroquinolone-treated rats (Kato et al., 1997; Shakibaei \& Stahlmann, 2001). The caspase and nitric oxide (NO) pathways play a role in tendon apoptosis as nitric oxide synthase (NOS) was found to be elevated in tendinopathy (Hashimoto $e t$ al., 1999). Treatment of tendon injuries and tendinopathies currently remains a clinical challenge, with repair often resulting in the production of inferior tissue and long-term complications and morbidity in patients. The need for treatment strategies that address the underlying pathophysiology of the damaged tissue is thus pertinent (Walden et al., 2017).The intrasound device produces mix frequency Intrasonic waves which are sound waves within the hearing threshold for humans $(16-20,000 \mathrm{~Hz})$. Some prior studies on acute tendon injury reported that intrasound therapy either in the low or high intensity mode resulted in significant proliferation of tenoblasts in the 
proliferative phase of healing with organized and advanced healing of the tendon by the $14^{\text {th }}$ day postinjury (Aiyegbusi et al., 2012), however, no prior study had been done on its effect on tendinopathy. Rats were used for this study because prior studies had established that both humans and rats exhibit common responses including lesions and edema to the use of fluoroquinolones, specifically Pefloxacin (PEF) (Szomo et al., 2001). The results of this study are important especially in managing FQ-induced tendinopathy in athletes who have been reported to use oral antibiotics twice as frequently as age-matched controls (2.7\% vs $1.3 \%$ ) (Alaranta et al., 2008). The purpose of this study was to determine and compare the effects of 7, 14 and 21 days intrasound therapy in improving the mechanical properties of the Achilles tendon in FQ-induced tendinopathy in animal models.

\section{MATERIAL AND METHODS}

Animals: The animals for this study were Fifty (50) male, 6 weeks old Sprague-Dawley rats purchased from the institutional Animal house. The animals were housed by five per wire-mesh cages with access to commercial pelleted rat chow and water ad libitum. Ethical approval was obtained from the institutional Health and Research Committee. All experimental procedures were performed in accordance with the National Institute of Health Guide for the Care and Use of Laboratory Animals (NIH Publications No. 8023 , revised in 1996).

Preparation of Pefloxacin: Pefloxacin (PEF) tablet $400 \mathrm{mg}$ was dissolved in distilled water and orally administered to the animals by gavage once daily for 6 consecutive days at a dose of $400 \mathrm{mg} / \mathrm{kg}$ of body weight in a fixed volume of $2.5 \mathrm{ml} / \mathrm{kg}$ body weight. A dosage of $400 \mathrm{mg}$ was chosen as a prior study had reported that PFX at a dose of $300 \mathrm{mg} / \mathrm{kg}$ caused lesions of the Achilles tendon (Kato et al., 1995).

Instrumentation: The Novasonic -Novafon Intrasonic device from Denmark was used for this study.

\section{Experimental Protocol}

Animal Grouping: Fifty (50) male rats that weighed between $100 \mathrm{gm}-120 \mathrm{gms}$ were randomized into the following groups:

Group 1 (Normal control): Animals were neither administered PEF nor ITR

Experimental control: All the animals were administered PEF solution by gavage at a dosage of $2.5 \mathrm{ml} / \mathrm{kg}$ body weight for 6 consecutive days. Group 2: (Experimental control): The animals were sacrificed on day 8 post PEF - (PEF0 8)
Group 3 (Experimental control): The animals were sacrificed on day 15 post PEF- (PEF015)

Group 4 (Experimental control): The animals were sacrificed on day 22 post PEF- (PEF0 22)

Treatment groups: Animals were administered PEF solution at a dosage of $2.5 \mathrm{ml} / \mathrm{kg}$ body weight by gavage for 6 consecutive days and afterwards commenced Intrasound therapy for 5 minutes once daily for the next consecutive 7, 14 and 21 days depending on the group Group 5: Animals had ITR for 7 days and were sacrificed of day 8, post ITR - (PI7 8)

Group 6: Animals had ITR for 7 days and were sacrificed of day 15, post ITR - (PI7 15)

Group 7: Animals had ITR for 7 days and were sacrificed of day 22, post ITR - (PI7 22)

Group 8: Animals had ITR for 14 days and were sacrificed of day 15, post ITR - (PI14 15)

Group 9: Animals had ITR for 14 days and were sacrificed of day 22, post ITR - PI14 22)

Group 10: Animals had ITR for 21 days and were sacrificed of day 22, post ITR - (PI 21 22)

Intrasound Therapy: The Achilles tendon of both hind limbs of each rat in the experimental groups were treated once daily with the lowest intensity of ITR using the small treatment head applied over the Achilles tendon for $5 \mathrm{~min}$ with sterile $\mathrm{K}-\mathrm{Y}$ jelly as a coupling medium (Potawski \& Watson, 2007). Treatment was given post- PFX administration for 7 , 14 and 21 consecutive days depending on the respective groups (Aiyegbusi et al., 2012).

Tendon Harvest Process: The animals were sacrificed and the Achilles tendons excised. The tendons were processed for histology using the technique of Stereology described by Young and Dyson (1990).

Stereological Analysis: The slides were observed under a light microscope fitted with an ocular test grid at a magnification of 400x using the method of CruzOrive and Weibel (1990). The tenocytes were identified by their nuclei and fifty random values (10 per animal) were obtained for each group. The numerical density $(N A)$ was calculated as the number of tenocyte profiles per unit area of field (Gundersen, 1986).

Estimation of Volume Fraction of Collagen: Estimation of collagen quantity was done in sections of tendon tissue embedded in paraffin and stained with hematoxylin and eosin (H\&E) as previously described (Avwioro, 2014). The volume fraction of the histological component was calculated as $V_{v}=P_{p} / P_{t}$, where $V_{v}$ is the volume fraction, $\mathrm{P}$ is the tissue component under consideration, and $\mathrm{Pp}$ is the number 
of test points associated with $\mathrm{P}$ and $\mathrm{Pt}$ the number of points of the test system (Aiyegbusi et al., 2012).

Preparation of Tendon Specimen for Biomechanical Evaluation: The tensile test specimen preparation was conducted in accordance with the American Standard testing and measurement, method D412 (ASTM D4121983). $50 \mathrm{~g}$ of epoxy and $25 \mathrm{~g}$ of hardener, which comprises of methyl ethyl ketone peroxide (MEKP) were used and the tendon immersed in the epoxy to form a tensile specimen (ASTM D412, 19830.

Biomechanical Testing: The tendon dimension of length $(28.5 \mathrm{~mm})$, width $(9.4 \mathrm{~mm})$, thickness $(4.3 \mathrm{~mm})$ and cross-sectional area (CSA) were measured three times using a digital image of ultrasonography (US). The specimen were tightly clamped on the material testing system of Universal Testing Machine (UTM) (Instron -Series 3369, Instron Corporation, Norwood, MA, USA) and subjected to static axial load at speed (strain rate) of $1 \mathrm{mms}^{-1}$ and tested according to established protocol (Davis, 2004). The different biomechanical properties were estimated according to established procedures.

Nitric Oxide Synthase Estimation: Nitric Oxidase Synthase (NOS) level was evaluated with prior documented protocol based on enzyme linked immunosorbent assay-double antibody sandwich principle (Nathan et al., 2007).

Statistical Analysis: The RSTUDIO version 4.0.02 was used to perform statistical analysis. Descriptive statistics of central tendency (mean, $M$ ) and dispersion (standard deviation, $S D$ ) were used to summarise the sample parameters in the control and experimental groups. Data normality in the Control and experimental groups were tested using Shapiro Wilk (Shapiro.wilk) normality test. The $\mathrm{R}$ programming language of ggpubr and ggplot2 (Alboukadel, 2020) were used to plot bar charts, do computation of oneway Analysis of Variance (ANOVA) and Kruskal Wallis rank sum. Post-hoc analysis of Tukey Honest Significant Difference (TukeyHSD) and Pairwise Wilcoxon Rank Sum Test (pairwise. Wilcox. test) were used for pairwise comparisons to identify mean(s) and median(s) that were different in the parameters of the experimental groups. Statistical level of significance of $5 \%$ was accepted. Pearson correlation was used to determine the linear relationship between NOS and the biomechanical and stereological properties in the experimental groups.

\section{RESULTS AND DISCUSSION}

The summary of data of the biomechanical properties of the Achilles tendon is presented in table1. There were no statistically significant differences (all $\mathrm{p}>$ 0.05) in the medians of all the biomechanical properties when the control and PEF only treated tendons were compared on days 8, 15 and day 22 . Similarly, there were no substantial differences (all $\mathrm{p}$ $>0.05)$ in the middle values of biomechanical properties between the PEF only and ITR groups on days 8, 15 and 22. Table 2 shows the percentage changes in biomechanical parameters when the PFx only groups were compared with the treatment groups at the different timelines of 8,15 and 22 days post treatment. The 7 days treatment had substantial improvement in the biomechanical parameters by the $8^{\text {th }}$ day though not significant statistically.

Table1: Biomechanical parameters of the Control and Experimental Groups

\begin{tabular}{|c|c|c|c|c|c|}
\hline Groups & $\begin{array}{l}\text { UTs (MPa) } \\
\text { M(SE) }\end{array}$ & $\begin{array}{l}\text { UTe }(\%) \\
\text { M(SE) }\end{array}$ & $\begin{array}{l}\text { Stiffness } \\
\text { (N/mm) } \\
\text { M(SE) }\end{array}$ & $\begin{array}{l}\text { E-M (GPa) } \\
\text { M(SE) }\end{array}$ & $\mathbf{L U s}(\mathbf{N}) \quad \mathbf{M}(\mathbf{S E})$ \\
\hline \multirow[t]{2}{*}{ Control } & $9.37(6.46)$ & $2.37(0.97)$ & $946.2(431.12)$ & $0.74(0.38)$ & $378.6(261.1)$ \\
\hline & \multicolumn{5}{|c|}{ Experiment A: PEF Only } \\
\hline P8 & $0.92(0.40)$ & $5.6(3.89)$ & $259.5(235.1)$ & $0.18(0.16)$ & $38.1(17)$ \\
\hline P15 & $2.95(1.87)$ & $2.63(0.57)$ & $397.8(106.5)$ & $0.28(0.08)$ & $119.2(75.6)$ \\
\hline \multirow[t]{2}{*}{$\mathbf{P 2 2}$} & $3.1(0.78)$ & $2.72(0.78)$ & $467.5(106.2)$ & $0.31(0.1)$ & $125.3(30.8)$ \\
\hline & \multicolumn{5}{|c|}{ Experiment B: PEF and Intra-sound } \\
\hline PI78 & $5.13(0.94)$ & $3.9(2.5)$ & $559.9(21.2)$ & $0.45(0.13)$ & $200(37.3)$ \\
\hline PI715 & $1.55(0.06)$ & $2.10(0.06)$ & $284.7(4.2)$ & $0.199(0.0)$ & $62.2(0.7)$ \\
\hline PI722 & $3.98(0.1)$ & $3.10(0.3)$ & $543.5(17.9)$ & $0.395(0)$ & $165.5(1.3)$ \\
\hline PI1415 & $2.39(0.05)$ & $2.22(0.26)$ & $519.8(165.3)$ & $0.366(0.12)$ & $96.6(8.1)$ \\
\hline PI1422 & $4.00(1.34)$ & $3.42(0.78)$ & $583.4(113.5)$ & $0.411(0.08)$ & $161.6(54.7)$ \\
\hline PI2122 & $3.49(0.44)$ & $2.11(0.31)$ & $645.8(124)$ & $0.455(0.09)$ & $141.1(17.7)$ \\
\hline
\end{tabular}

UTs = Ultimate Tensile Stress, UTe = Ultimate Tensile Strain, E-M = Elastic (Young's) Modulus, LUs = Load at Ultimate Stress, $P 8=P E F$ only 7 days euthanized day 8,P15=PI78 =EF, Intra-sound treated for 7 days, euthanized day 15, P22=PEF only for 7 days, euthanized day 22

PI78 =PEF, Intra-sound treated for 7 days, euthanized day 8, PI715 = PEF, Intra-sound treated for 7 days, euthanized day 15, PI722= $P E F$, Intra-sound treated for 7 days, euthanized day 21

PI1415 = PEF, Intra-sound treated for 14 days, euthanized day 15, PI14 $22=$ PEF, Intra-sound treated for 14 days, euthanized day 22 PI2122 = PEF, Intra-sound treated for 21days, euthanized day 22. 
Table 2: Changes in Selected Biomechanical Parameters in Timelines across the Pefloxacin Only and Intrasound Treatment Groups

\begin{tabular}{|c|c|c|c|c|c|c|}
\hline \multirow[b]{2}{*}{ Day 8} & \multirow[b]{2}{*}{$\begin{array}{l}\text { P8 } \\
\text { PI78 }\end{array}$} & $\mathrm{UT}_{3}$ & $\mathrm{UTe}$ & Stiffness & E-M & $\mathrm{LU}_{3}$ \\
\hline & & $\begin{array}{l}+458 \% \\
p=0.2\end{array}$ & $\begin{array}{l}-30.4 \% \\
p=0.2\end{array}$ & $\begin{array}{l}+116 \% \\
p=0.2\end{array}$ & $\begin{array}{l}+150 \% \\
p=0.2\end{array}$ & $\begin{array}{l}+425 \% \\
p=0.2\end{array}$ \\
\hline \multirow[t]{2}{*}{ Day 15} & $\begin{array}{l}\text { P15 } \\
\text { PI715 }\end{array}$ & $\begin{array}{l}-47.5 \% \\
p=0.66\end{array}$ & $\begin{array}{l}-20.2 \% \\
p=0.66\end{array}$ & $\begin{array}{l}-28.4 \% \\
p=0.66\end{array}$ & $\begin{array}{l}-29 \% \\
p=0.66\end{array}$ & $\begin{array}{l}-47.8 \% \\
p=0.66\end{array}$ \\
\hline & $\begin{array}{l}\text { P15 } \\
\text { PI1415 }\end{array}$ & $\begin{array}{l}-19 \% \\
p=0.66\end{array}$ & $\begin{array}{l}-16 \% \\
p=0.82\end{array}$ & $\begin{array}{l}+30.7 \% \\
p=0.66\end{array}$ & $\begin{array}{l}+30.7 \% \\
p=0.66\end{array}$ & $\begin{array}{l}-23.4 \% \\
p=0.66\end{array}$ \\
\hline & $\begin{array}{l}\mathrm{P} 22 \\
\mathrm{P} 722\end{array}$ & $\begin{array}{l}+28.4 \% \\
p=0.095\end{array}$ & $\begin{array}{l}+14 \% \\
p=1\end{array}$ & $\begin{array}{l}+16.3 \% \\
p=1\end{array}$ & $\begin{array}{l}+27.4 \% \\
p=1\end{array}$ & $\begin{array}{l}32.1 \% \\
p=0.095\end{array}$ \\
\hline Day 22 & $\begin{array}{l}\text { P22 } \\
\text { PI1422 }\end{array}$ & $\begin{array}{l}+29 \% \\
p=0.67\end{array}$ & $\begin{array}{l}+25.7 \% \\
p=0.67\end{array}$ & $\begin{array}{l}+24.8 \% \\
p=0.6\end{array}$ & $\begin{array}{l}+32.6 \% \\
p=0.67\end{array}$ & $\begin{array}{l}+29 \% \\
p=0.67\end{array}$ \\
\hline & $\begin{array}{l}\text { P22 } \\
\text { PI2122 }\end{array}$ & $\begin{array}{c}+12.6 \% \\
p=0.8\end{array}$ & $\begin{array}{l}-22.4 \% \\
p=0.53\end{array}$ & $\begin{array}{c}+38.1 \% \\
p=0.53\end{array}$ & $\begin{array}{l}+46.8 \% \\
p=0.53\end{array}$ & $\begin{array}{c}+12.6 \% \\
p=0.8\end{array}$ \\
\hline
\end{tabular}

UTs = Ultimate Tensile Stress, UTe = Ultimate Tensile Strain, E-M = Elastic (Young's) Modulus, LUs= Load at Ultimate Stress, $P 8=P E F$ only 7 days euthanized day 8, P15 =PI78 =EF, Intra-sound treated for 7 days, euthanized day 15, $P 22=P E F$ only for 7 days, euthanized day 22

$P I 78=P E F$, Intra-sound treated for 7 days, euthanized day 8, PI715 = PEF, Intra-sound treated for 7 days, euthanized day 15, PI722= $P E F$, Intra-sound treated for 7 days, euthanized day 21

PI1415 = PEF, Intra-sound treated for 14 days, euthanized day 15, PI14 $22=$ PEF, Intra-sound treated for 14 days, euthanized day 22 PI2122 = PEF, Intra-sound treated for 21days, euthanized day 22.

Table 3: Biochemical and Stereological Parameters of the Control and Experimental Groups

\begin{tabular}{llll}
\hline Group & $\begin{array}{l}\text { NOS }(\boldsymbol{\mu m o l e s} / \mathbf{l}) \\
\text { M(SD) }\end{array}$ & $\begin{array}{l}\text { Tenocyte count } \\
\text { /unit area M(SD) }\end{array}$ & $\begin{array}{l}\text { \% Vol Fraction of } \\
\text { Collagen M(SD) }\end{array}$ \\
\hline Control & $17.19(8.42)$ & $38.3(16.20$ & $6.21(0.69)$ \\
PEF08 & $27.39(10.82)$ & $33.6(11.9)$ & $5.56(3.21)$ \\
PEF015 & $12.09(3.9)$ & $38.7(6.3)$ & $6.36(4.17)$ \\
PEF021 & $17.44(2.52)$ & $40.2(7.8)$ & $6.52(3.05)$ \\
PEF78 & $12.32(8.16)$ & $34.8(11.2)$ & $5.7(1.12)$ \\
PEF715 & $3.59(2.55)$ & $44.7(8.6)$ & $7.34(4.28)$ \\
PEF721 & $15.06(8.4)$ & $40.2(16.6)$ & $6.5(3.21)$ \\
PEF1415 & $10.06(6.67)$ & $49.7(10.3)$ & $8.17(1.41)$ \\
PEF1421 & $14.64(8.42)$ & $45.3(7.2)$ & $7.48(0.15)$ \\
PEF2122 & $11.24(1.2)$ & $53.6(11.6)$ & $8.8(4.32)$ \\
p-value & 0.0744 & 0.774 & 0.9684 \\
\hline
\end{tabular}

$P 8=P E F$ only 7 days euthanized day $8, P 15=P I 78=E F$, Intra-sound treated for 7 days, euthanized day $15, P 22=P E F$ only for 7 days, euthanized day 22

$P I 78=P E F$, Intra-sound treated for 7 days, euthanized day 8, PI715 = PEF, Intra-sound treated for 7 days, euthanized day 15, PI722= $P E F$, Intra-sound treated for 7 days, euthanized day 21

$P I 1415=P E F$, Intra-sound treated for 14 days, euthanized day 15, PI14 $22=$ PEF, Intra-sound treated for 14 days, euthanized day 22 PI2122 = PEF, Intra-sound treated for 21days, euthanized day 22.

The 14 days treatment appeared to have more sustainable effects and by day 22 as seen in figures 1 and 2 , there were no significant differences in the biomechanical variables among the PEF only and treatment groups. The summary of the biochemical and stereology data in mean (M) and standard error (SE) is presented in table 3 . There were no statistically significant differences (all $\mathrm{p}>0.05$ ) in the medians of the biochemical and stereology parameters of NOS, tenocyte population and volume fraction of collagen when the control and experimental groups were compared. On table 4 is shown the correlations between NOS expression and the different variables evaluated.
In the PEF only groups, all the variables had negative correlations with NOS except the Ultimate tensile strain which had significant strong positive correlation. In the Intrasound groups on the other hand, there are significant and strong positive correlations of NOS with ultimate tensile stress and tensile load and moderate negative association with the tenocyte population while there are weak insignificant negative correlations with the ultimate strain and volume fraction of collagen. Disorders of the Achilles tendon rank among the most frequently reported overuse injuries and have been reported to afflict both athletes and sedentary persons (Huttunen et al., 2014). This has been worsened by the popular 
use of fluoroquinolones to treat infections because the use of this brand of antibiotics has been linked to a number of adverse effects, including FQ-associated tendinopathy which was first reported in literature in 1983 (Bailet et al., 1983). This study is pertinent because it has been reported that several cases of FQassociated tendinopathy involve sporting or very physically active people due to the combined adverse effects of exercise and regular use of fluoroquinolones even as athletes have been found to use oral antibiotics twice as frequently as age-matched controls $(2.7 \%$ vs 1.3\%) (Ng and Naughton, 2007; Alaranta, 2008; Lewis, 2009). The choice of PEF for this study was premised on the fact that it is categorized as one of the newer FQs found to be more more toxic for cartilages and tendons while also being one of the most frequently prescribed antibiotic (Kashida and Kato, 1997; Jeffrey \& Aronson, 2016). The treatment of tendon disorders, particularly tendinopathies have been challenging clinically with the healing tendon having biomechanical properties which are inferior to the normal tendon, thereby posing a risk of re-injury and subsequent tendon rupture. Moreover, it has been reported that recovery from fluoroquinolone-related tendinopathy is sometimes slower than from other types of tendinopathy (Trevor \& Cook, 2014). It becomes pertinent therefore to investigate modalities that may augment healing with better biomechanical properties even as it has been earlier reported that the closer the Elastic modulus of a tendon is to the normal value, the more it will respond normally under loading (Khayyeri et al., 2017).

Table 4: Correlation of NO with Biomechanical and Morphological Variables

\begin{tabular}{|c|c|c|}
\hline Biomechanical Properties & PEF Only & PEF and INTRASOUND \\
\hline & (r, p-value) & (r, p-value) \\
\hline Ultimate Stress vs. NOS & $-0.46,0.297$ II & $0.62,0.005 * *+$ \\
\hline Ultimate Strain vs. NOS & $0.86,0.012 * * \dagger$ & $-0.01,0.978$ \\
\hline Maximum Load vs. NOS & $-0.46,0.297$ | & $0.64,0.003 * *+$ \\
\hline Stiffness vs. NOS & $-0.73,0.065 \dagger$ & $0.29,0.232$ \\
\hline Young's Modulus vs. NOS & $-0.74,0.06 \dagger$ & $0.26,0.278$ \\
\hline Biochemical \& Stereology Prop & & \\
\hline$\%$ Vol. of Collagen vs. NOS & $-0.36,0.427$ II & $-0.25,0.301$ \\
\hline Tenocyte Count/Area vs. NOS & $-0.67,0.102 \ddagger$ & $-0.56,0.012 * *+$ \\
\hline
\end{tabular}

This study was therefore carried out as part of an ongoing attempt to investigate modalities that could improve and reverse the biomechanical and morphological changes seen in tendinopathy. Intrasound therapy has been reported by prior studies to augment and improve the morphological properties of the healing tendon following acute tendon injury (Aiyegbusi et al., 2012). Though no study has been done on its effect on the biomechanical properties in tendinopathies, hence the need for this study. Prior studies had established that both humans and rats exhibit common responses including lesions and edema to the use of fluoroquinolones, specifically Pefloxacin (Kashida \& Kato, 1997). However, it has been reported that the injection of fluoroquinolones in rats does not lead to tendon rupture as it does in humans (Ribard et al., 1992) and a lower dosage in humans seems to cause more severe effects than in rats, even as Kato et al., (1997) had demonstrated that a single oral dose of fluoroquinolones in juvenile rats induced reversible lesions 2 weeks after administration. Going by this assertion, it is expected that the $400 \mathrm{mg} / \mathrm{kg}$ dosage used in this study will have more severe effect in humans, especially as PEF is usually used at a higher daily dosage of $800 \mathrm{mg}$ to treat infections in humans and the majority of cases of tendinopathy occurred with a PEF dosage of $800 \mathrm{mg}$ per day (Meyboom et al., 1994; Khaliq and Zhanel, 2003).

The results of this study showed that the tendons that had PEF administered without treatment had their mechanical integrity compromised by day 8 post-PEF even as a prior study had reported 6 days as the median onset of tendinopathy following FQ treatment (Corrao et al 2006). PEF treatment has been reported to induce oxidative damage of type 1 collagen by direct toxicity (Van der Linden et al., 2001; Pouzaud et al., 2004). The altered biomechanical properties of the PEF - only treated tendons was evident by the highest ultimate tensile strain and reduced stiffness observed in this group especially on the $8^{\text {th }}$ day post PEF while the other biomechanical properties were much reduced when compared with the control, though the changes were not statistically significant. The statistically insignificant changes in the variables in the experimental groups in spite of the evident biological changes are likely due to the relatively small sample sizes used in this study. It was however observed that by day 22 Post PFx, these properties had significantly improved when compared with the values on days 8 and 15 post-PFx. A prior study had reported that lower dosage in humans seems to cause more severe effects than in rats which could explain the fact that 
tendinopathic lesions seen were not too pronounced and by day 22, there was ample recovery as seen in table 2 where all the biomechanical properties had improved by then, though with inferior biomechanical properties when compared with the normal tendon. Further studies are needed with much higher dosage, such as $800 \mathrm{mg} / \mathrm{kg}$ body weight as given in clinical settings. Also, the expression of NOS was highest in the PEF-only group on day 8 post-PEF to stimulate extracellular matrix synthesis as reported in earlier studies that the expression of NO is up-regulated in tendinopathy to enhance and promote collagen synthesis with a return to baseline by the $14^{\text {th }}$ day (Xia et al., 2006; Murrell, 2007). Our results show a negative correlation between the biomechanical properties and NO expression in the PEF only group except for the Ultimate tensile strain which showed a significant $(p<0.05)$ high correlation. This may be indicative of the fact that NO is normally not regulated in a normal tendon so as the biomechanical properties are altered, NO is increased to stimulate healing, even as it seen in table 3 that the expression of NO was highest on the $8^{\text {th }}$ day Post-PEF (Xia et al., 2006). The need for modalities that will augment healing in tendinopathies cannot be over-emphasized as significant structural changes have been shown to persist in animal studies for as long as 20 weeks post FQ exposure with clinical symptoms reported to manifest as late as 6 months after exposure (Khaliq and Zhanel, 2003; Shakibaei et al., 2007).This could make sportsmen and physically active individuals prone to re-injury and the risk of tendon rupture.
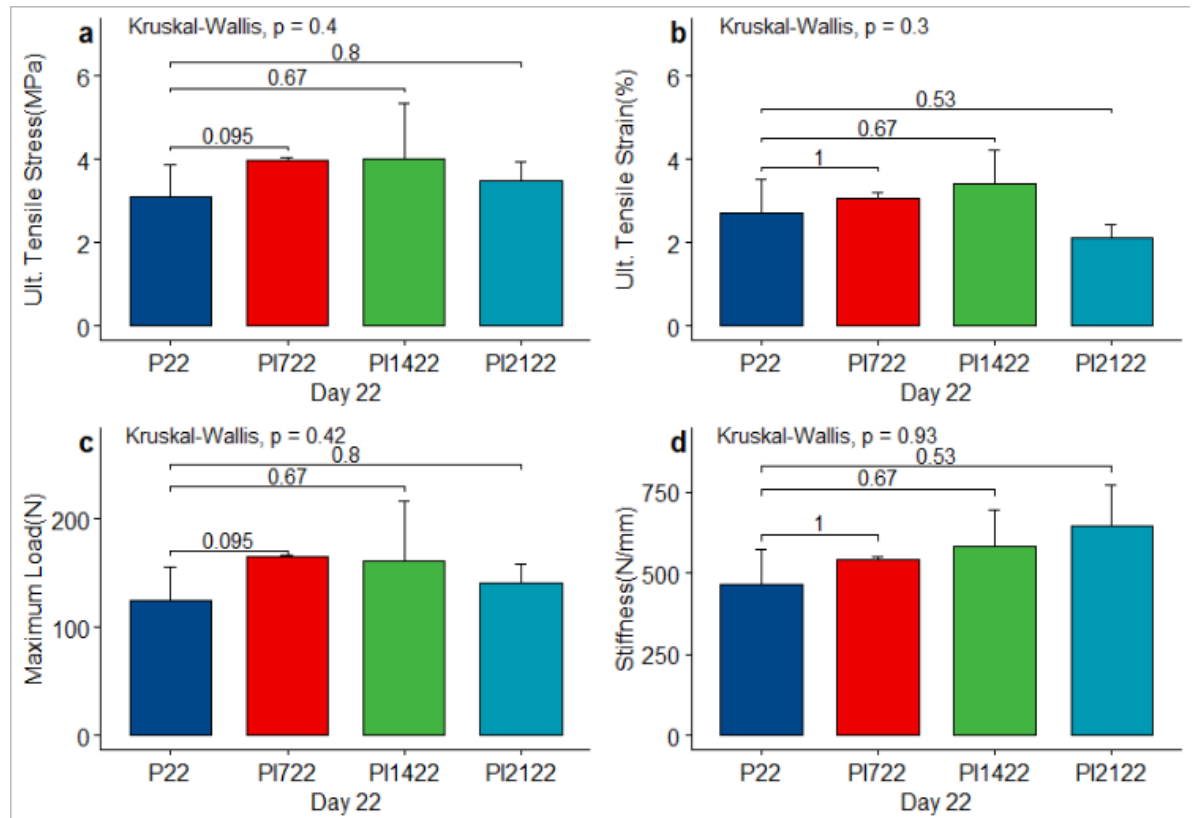

Fig 1: Comparisons of day 22 effects in biomechanical properties of the Achilles tendon.
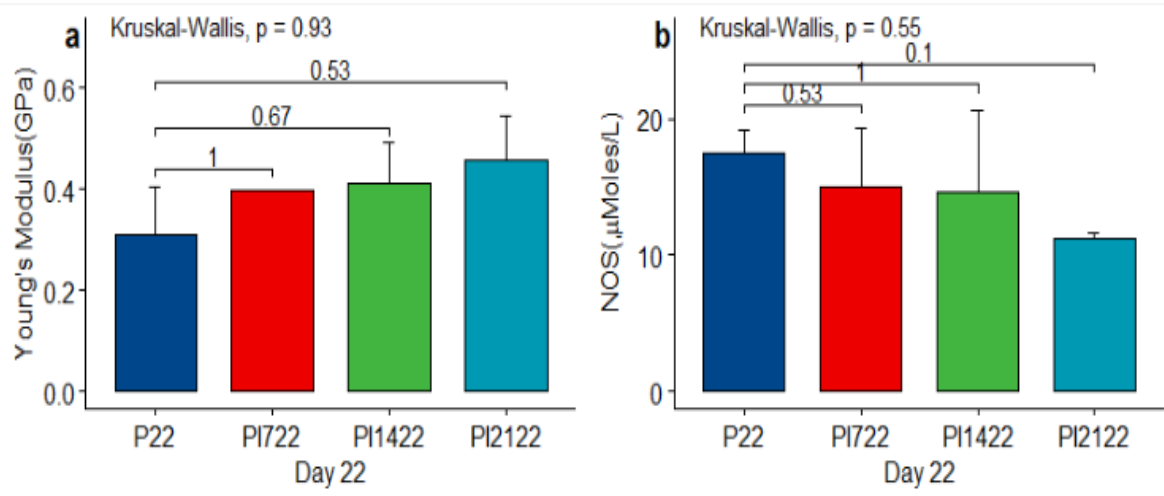

Fig 2: Comparisons of day 22 effects of Young's modulus and NOS of the Achilles tendon. 
The animals that had intrasound treatment post-PFx exhibited varied responses as seen from our results. The 7 day- treatment showed markedly improved biomechanical parameters by day 15 post -PFX which however were reduced, though not significantly by day 22. As seen in table 2, there was significant $(p<0.05)$ increase in all the biomechanical variables analysed in the tendons in this group when compared with the experimental control. However, the effects of the 7-day treatment was not sustainable till day 22 . The 14 days treatment on the other hand had the best biomechanical qualities by the end of the $22^{\text {nd }}$ day when compared with the PFx only tendons, though these qualities were still inferior to the normal tendons. The Young modulus (E-M) is described as a measure of material resistance (stiffness) to elastic deformation under load and the closer the elastic modulus of a loaded tendon is to that of a control experiment, the more similarly it will behave under mechanical stress (Khayyer, 2017). Our results showed that the 14 and 21 days treated tendons had the nearest E-M to the normal tendon. The inferior mechanical properties seen in the PEF only group can also be seen as markedly reduced stiffness of the tendons. Stiffness, which is an important constituent of tendon mechanical properties has a significant influence on force transmission as an optimal level of tendon stiffness is critical for effective muscle-tendon interactions (Bojsen-Moller et al., 2005; Reeves et al., 2003). Our results show that by day 22, the tendons treated for 14 and 21 days had the closest value of stiffness to the control group. However, from figures $1 \& 2$ which showed the biomechanical properties of the tendons across the groups on day 22 , it can be seen that the 14 days treatment group had the best biomechanical properties.

The significant positive correlations between NOS expression and some of the biomechanical properties in the intrasound group may suggest that ITR regulates NOS expression during tendon healing which may be responsible for the improved properties seen in this group as it has been previously reported that the addition of $\mathrm{NO}$ had a protective or beneficial effect on collagen organisation, tendon healing failure load and stress (load/area) (Murrell et al., 1997; Bokhari \& Murrell, 2011). Comparing the tenocyte population and Volume fraction of Collagen across the control and experimental groups show no significant differences ( $\mathrm{p}>0.05)$. This is expected as it has been reported that tendinopathic tendons are thicker, but with reduced energy-storing capacity, which implies that the tendons exhibit higher strains for the same load than healthy tendons thus exhibiting a decline in both the structural and material properties, as reported also by a prior study (Helland et al., 2013;
Alice et al., 2014). This is seen from our results where the Ultimate tensile stress of the PEF tendon on day 8 was about a tenth of the normal value, while the volume fraction of collagen was almost the same as the control group. However, the quality and types of collagen fibres in the various treatment groups were not ascertained in this study even as prior studies have demonstrated an increase in volume and size of tendon tissue in tendinopathy but with inferior mechanical properties due to increase production of type 3 collagen fibres with small diameters over type 1 which is synthesized in abundance during the repair process in degenerative tendons (Chan et al., 2008; Thorpe et al., 2015). This is pertinent as a prior study had reported a relationship between different collagen fibril diameters in tendons and variations in tendon strength and elasticity (Biancalana et al., 2010). Since the remodelling phase of tendon healing which begins from day 21 is characterized by greater structural organization, we hypothesize that the tendons treated with ITR could demonstrate greater biomechanical resistance in the later stages of the repair process. Further studies are recommended to evaluate the effects of ITR in the later stages of this phase.

Conclusion: Intrasound treatment to the Achilles tendon for 14 days improved the mechanical properties and morphology of tendons in Fluoroquinolone-induced tendinopathy probably by regulating the expression of NOS.

Acknowledgements: We appreciate Prof O.A Adeosun for his assistance with the biomechanical analysis and: the support by Tertiary Education Fund (TETFUND) through a grant with Number TETFUND IBR GRANT- CRC/TETEFUND/NO. 2018/01

\section{REFERENCES}

Aiyegbusi, AI; Duru, FIO; Akinbo, SRA (2012). The morphology of the healing tendon: A comparison of the effects of Intrasound therapy and therapeutic pulsed Ultrasound. Connect Tissue Res. 53 (6): 478-484

Alaranta, A,;Alarenta, H; Helenius, I. (2008) Use of prescription drugs in athletes. Sports Med. 38:449463.

Alice, JS. Fox, Michael O. Scha; Florian Wanivenhaus; Tony Chen; Erik Attia; Nikolaus B. Binder (2014) .Fluoroquinolones Impair Tendon Healing in a Rat Rotator Cuff Repair Model. The Am J Sports Med. 42. 12 
Alboukadel Kassambara. ggpubr: 'ggplot2' Based Publication Ready Plots. R package version 0.4.0. https://cran.r-project.org/package=ggpubr c2020.

Aranha O; Wood DP J; Sarkar FH. (2000). Ciprofloxacin mediated cell growth inhibition, S/G2-M cell cycle arrest, and apoptosis in a human transitional cell carcinoma of the bladder cell line. Clin Cancer Res. Mar; 6(3):891-900.

ASTM D412. (1983). Standard Test Methods for Vulcanized Rubber and Thermoplastic ElastomersTension. Amer Soc for Testing and Materials.

Avwioro G 92014). Histochemistry and tissue pathology, principles and techniques. $3^{\text {rd }} \mathrm{Ed}$. Ibadan, Nigeria: Claverianun Press p155.

Bailey, RR; Kirk, JA; Peddie, BA (1983). Norfloxacin-induced rheumatic disease. New Zealand Med J. 96(736):590

Biancalana, A; Veloso, LA;Gomes, L (2010). Obesity affects collagen fibril diameter and mechanical properties of tendons in Zucker rats. Connect Tissue Res. 51(3), 171-178.

Bokhari, AR; Murrell, GA (2012). The role of nitric oxide in tendon healing. J Shoulder Elbow Surg 21:238-44.

Bojsen-Moller, J; Magnusson, SP; Rasmussen, LR; Kjaer, M; Aagaard ,P (2005).. Muscle performance during maximal isometric and dynamic contractions is influenced by the stiffness of the tendinous structures. J Appl Physiol. 99: 986-994

Budny, AM; Ley, AN (2015).Fluoroquinolonemediated Achilles rupture: A case report and review of the literature.J Foot and Ankle Surg. 54(3):494-496.

Chan, $\mathrm{KM}$; $\quad \mathrm{Fu}, \quad \mathrm{SC} ; \quad$ Wong, YP;, Hui,W.C; Cheuk,YC; Wong, MW (2008). Expression of transforming growth factor beta isoforms and their roles in tendon healing. Wound Repair Regen. 16, 399

Childs, SG (2007).. Pathogenesis of tendon rupture secondary to fluoroquinolone therapy. Orthop Nurs. 26:175-182.

Corrao. G; Zambon, A; Bertù, L; Mauri, A; Paleari,V; Rossi, C; Venegoni, M (2006). Evidence of tendinitis provoked by fluoroquinolone treatment: a case-control study. Drug Saf. 29(10):889-96.
Cruz-Orive, LM; Weibel, ER (1990). Recent stereological methods for cell biology brief survey. Am. J. Physiol. 258:L148-L156.

Davis, Joseph R (2004). Tensile testing. 2nd ed. ASM International p 2

Desbordes, JM; Bouquet, S; Breux, J; Chapelle, G; Bataille, B; Becq, Giraudon B; Fusciardi, J (1993). Comparative penetration of 3 fluoroquinolones into human cranial bone tissue after administration of a single intravenous dose. Drugs. 45(Suppl. 3):448-449.

Grace Walden; Xin Liao; Simon Donell; Mike J. Raxworthy; Graham P. Riley; Aram Saeed. A (2017). Clinical, Biological, and Biomaterials Perspective into Tendon Injuries and Regeneration. Tissue Eng Part B: Rev. Feb;23(1):44-58.

Gundersen, HJG (1986). Stereology of arbitrary particle: A review of unbiased number and size estimators and the presentation of new ones; in memory of William R Thompson. J. Microsc. $143: 3-45$.

Hashimoto, S; Takahashi, K; Ochs, RL; Coutts, RD; Amiel D; Lotz M (1999). Nitric oxide production and apoptosis in cells of the meniscus during experimental osteoarthritis. Arthritis Rheum 42:2123-2131.

Helland, C; Bojsen-Møller,J; Raastad, T (2013). Mechanical properties of the patellar tendon in elite volleyball players with and without patellar tendinopathy. Br J Sports Med. 47:862-868.

Huttunen, TT; Kannus, P; Rolf, C; Felländer-Tsai, L; Mattila,VM (2014). Acute achilles tendon ruptures: incidence of injury and surgery in Sweden between 2001 and 2012. Am J Sports Med. 42(10):2419-2423.

Jeffrey, K; Aronson, JK (2016). Meyler's side effects of drugs. Amsterdam: Elsevier Science, 16th Ed.

Kashida, Y; Kato, M (1997). Characterization of fluoroquinolone-induced Achilles tendon toxicity in rats: comparison of toxicities of 10 fluoroquinolones and effects of anti-inflammatory compounds. Antimicrob Agents Chemother. 41:2389-2393.

Kato, M S, Takada, Y; Kashida; M.(1997). Histological examination on Achilles tendon 
lesions induced by quinolone antibacterial agents in juvenile rats. Toxicol. Pathol. 23:385-392.

Khaliq,Y, Zhanel, GG (2003). Fluoroquinoloneassociated tendinopathy: a critical review of the literature. Clin Infect Dis ;36:1404-1410.

Khayyeri, H; Blomgran, P; Hammerman M; Turunen, M.J; Lowgren, A, Guizar-Sicairos et al.,(.2017). Achilles Tendon Compositional and Structural Properties are Altered after Unloading by Botox. Scientific Reports,7:13067, doi:10.1038/s441598017-13107-7.

Lewis, TG (2009). A rare case of ciprofloxacininduced bilateral rupture ofthe Achilles tendon.BMJ Case Rep. doi:10.1136/bcr.08.2008.0697.

Meyboom, RHBS; Olsson, A; Knol, J; Dekens-Konter, AM; De Koning GHP (1994). Achilles tendinitis induced by peflfloxacin and other fluoroquinolone derivatives. Pharmacoepidemiol. Drug Safety.3:185-189.

Murrell George A.C . Using nitric oxide to treat tendinopathy. BJSM 2007;41:227-231.

Murrell GAC, Szabo C, Hannafin JA, et al. Modulation of tendon healing by nitric oxide. Inflamm Res 1997;46:19-27

Nathan, S. Bryan; Matthew B. Grisham (2007). Methods to Detect Nitric Oxide and its Metabolites in Biological Samples. Free Radic Biol Med. 43(5): 645-657.

$\mathrm{Ng}$, WF (2007). Naughton M. Fluoroquinoloneassociated tendinopathy: a case report.J Med Case Rep.;1:5

Potawski, L; Watson, $\mathrm{T}$ (2007). Relative transmissivity of ultrasound coupling agents commonly used by therapist in the UK. Ultrasound Med. Biol. 33(1):120-128.

Pouzaud F; Bernard-Beaubois K; Thevenin M; Warnet JM; Hayem G (2004). Rat P. In vitro discrimination of fluoroquinolones toxicity on tendon cells: involvement of oxidative stress. $J$ Pharmacol Exp Ther. 308: 394-402.

Reeves, ND; Maganaris, CN; Narici,MV (2003). Effect of strength training on human patella tendon mechanical properties of older individuals. J Physiol. 548: 971-981
Ribard, P; Audisio, F; Kahn, MF; De Bandt, M; Jorgensen, C; Hayem, G (1992).. Seven Achilles tendinitis including 3 complicated by rupture during fluoroquinolone therapy. $J$ Rheumatol 19(9):1479-81.

Shakibaei, M; Stahlmann, R. (2001). Ultrastructure of Achilles tendon from rats after treatment with fleroxacin. Arch Toxicol . 75(2):97-102.

Seeger, JD; West, WA; Fife, D; Noel, GJ; Johnson, LN; Walker, AM (2006). Achilles tendon rupture and its association with fluoroquinolone antibiotics and other potential risk factors in a managed care population. Pharmacoepidemiol Drug Saf. 15:784792

Sode, J; Obel, N; Hallas, J; Lassen, A (2007). Use of fluoroquinolone and risk of Achilles tendon rupture: a population-based cohort study. Eur $J$ Clin Parmacol. 63:499-503.

Szomor, L; Wang, MX; Kruller, A (2001). Differential expression of cytokines and nitric oxide synthase isoforms in human rotator cuff bursae. Ann Rheum Dis. 60:431-2.

Stephenson, AL; Wu, W; Cortes, D; Rochon, PA (2013). Tendon injury and fluoroquinolone use: A systemic review. Drug Saf. 36:709-721.

Thorpe, CT; Spiesz, EM; Chaudhry,S; Screen, HRC; Clegg, PD (2015). Science in brief: recent advances into understanding tendon function and injury risk. Equine Vet J. 47, 137

Trevor, L; Cook, J (2014). Fluoroquinolones and Tendinopathy: A Guide for Athletes and Sports Clinicians and a Systematic Review of the Literature.J Athl Train. 49(3):422-427

Van der Linden, PD; van Puijenbroek, EP; Feenstra, J.(2001). Tendondisorders attributed to fluoroquinolones: a study on 42 spontaneousreports in the period 1988 to 1998. Arthritis Rheum. 45(3):235-239.

Xia, W; Szomor, Z; Wang,Y (2006). Nitric oxide enhances collagen synthesis in cultured human tendon cells. J Orthop Res. 24:159-72

Young, SR, Dyson, M (1990). The effect of therapeutic ultrasound on the healing of full thickness excised skin lesions. Ultrasonics. 28: 175-180 